\title{
Whole Blood Fatty Acid Composition Differs in Term Versus Mildly Preterm Infants: Small Versus Matched Appropriate for Gestational Age
}

\author{
CARLO AGOSTONI, FRANCA MARANGONI, GIORGIO STIVAL, ITALO GATELLI, FRANCESCA PINTO, PATRIZIA RISÉ, \\ MARCELLO GIOVANNINI, CLAUDIO GALLI, AND ENRICA RIVA
}

Department of Pediatrics [C.A., G.S., I.G., F.P., M.G., E.R.], University of Milan, I-20142 Milan, Italy; Department of Pharmacological Sciences [F.M., P.R., C.G.], University of Milan, I-20133 Milan, Italy

\begin{abstract}
To investigate the associations between whole blood fatty acid (FA) profile and restricted intrauterine growth, any small for gestational age (SGA) infant born in our maternity ward through $1 \mathrm{y}$ was matched with two appropriate for gestational age (AGA), of the same GA $\pm 0.5 \mathrm{wk}$, infants, further subdivided into term and preterm. Whole blood was collected at d 4 on a strip and FA \% composition assessed by means of gas chromatography. The whole sample consisted of 28 SGA versus 56 AGA born at term and 20 SGA versus 40 AGA born preterm at around 35 wks. Parent FA of the n- 6 and n- 3 FA families were higher in preterm groups, whereas docosahexaenoic acid was higher in term AGA (median \% values, 3.9 versus 3.7 in term SGA, 2.8 in preterm AGA, and 2.5 in preterm SGA, $p<0.001)$. Term AGA had markedly higher values for the docosahexaenoic acid/alpha-linolenic acid ratio (median value: 91, versus 18 in term SGA, 12 in preterm AGA, and 10 in preterm SGA, $p<0.001)$. Term SGA had significantly lower levels of total monounsaturated FA and higher levels of eicosapentaenoic acid. Therefore, the 4-d whole blood FA pattern is associated with both GA and birth weight. (Pediatr Res 64: 298-302, 2008)
\end{abstract}

$\mathrm{L}$ ong chain polyunsaturated fatty acids (LCPUFAs) of the n-6 and n-3 series are metabolically derived from the essential 18 carbon $n-6$ and $n-3$ precursors, linoleic acid (LA, 18:2 n-6) and alpha-linolenic acid (ALA, 18:3n-3), and play major roles in early development, being deeply involved in neurodevelopment and generally in growth processes (1).

Most of the n-3 and n- 6 LCPUFA are acquired by the fetus in utero across the placenta (2). The pattern of LCPUFA transferred from the mother to the fetus depends on maternal stores, maternal dietary intakes, and metabolic processes taking place in the placenta (3). By the end of the second trimester of pregnancy, the fetus is capable to directly synthesize LCPUFA from their precursors (4). In the last weeks of pregnancy, a biomagnification process takes places in mammalian species, leading to a preferential accumulation of the LCPUFA derivatives in the fetus (5). Therefore, prematurity directly reduces the LCPUFA bioavailability (6).

Within term and preterm infants, intrauterine growth restriction (IUGR) is a condition associated with failure of the placenta to provide the necessary nutrients required by the fetus to maintain adequate growth. This is shown by the observation that in

Received February 14, 2008; accepted April 18, 2008.

Correspondence: Carlo Agostoni, M.D., Department of Pediatrics, San Paolo Hospital, 8 Via A. di Rudinì, I-20142 Milan, Italy; e-mail: carlo.agostoni@unimi.it both the n-3 and n-6 PUFA families, the proportions of the long-chain derivatives, arachidonic acid (AA, 20:4 n-6) and docosahexaenoic acid (DHA, 22:6 n-3), are lower versus an elevation of their precursors, LA and ALA, respectively, in cases of IUGR (7). As result of IUGR due to reduction or an arrest of growth processes at various stages during the fetal life, infants at birth are small for gestational age (SGA), that is, below the 10th percentile for expected weight, compared with infant counterparts with birth weight appropriate for gestational age (AGA).

Studies with stable isotopes have shown that growth retardation slows down or reduces LCPUFA formation (8), the synthesis of n-3 compounds being more extensively affected (9). The evaluation of the LCPUFA status is therefore relevant to appreciate physiologic processes and establish dietary needs of preterm and SGA infants, respectively. Studies so far have compared LCFUFA levels of AGA versus SGA infants in a very wide interval of gestational age in utero (7), or at birth by analyzing umbilical cord plasma phospholipids within a large population inclusive of a small percentage of SGA infants (10). Also, in studies with isotopic tracers, heterogeneous groups have been progressively considered, that is, mildly preterm SGA (around $36 \mathrm{wks}$ ) versus preterm AGA (30 wks) versus term AGA (8), and mildly preterm SGA (34 wks) versus mild preterm AGA (34 wks) versus preterm AGA (around $30 \mathrm{wks}$ ) (9), based on comparisons of the study group with infants of comparable GA or weight, respectively. No study has also considered associations with maternal dietary intakes throughout pregnancy.

The aim of this investigation was studying four groups, healthy term and mildly preterm infants, further subdivided into AGA and SGA per group, within a matched design by means of the determination of the fatty acid (FA) pattern on whole blood (inclusive of all circulating lipid fractions and cells) collected on a special strip of adsorbent at d 4 on occasion of the Guthrie test, and controlling for maternal dietary habits in pregnancy. Sampling of infant's blood was therefore carried out at a very early age, comparable with

Abbreviations: AA, arachidonic acid; AGA, Appropriate for gestational age; ALA, Alpha-linolenic acid; DHA, Docosahexaenoic acid; EPA, Eicosapentaenoic acid; FA, Fatty acid; LA, Linoleic acid; LCPUFA, Long-chain polyunsaturated fatty acids; SGA, Small for gestational age 
sampling of cord blood at birth, but eliminating the possible contamination with maternal blood (11). This minimally invasive method for whole blood FA analysis not only facilitates the collection of samples, but also allows their easy storage and shipment to the analytical laboratories. In most LCPUFA studies, blood samples are separated and plasma and red cell PUFA values reported separately. The whole blood collected with this method represents the available "pool" of LCPUFA, with relevance for human studies on a large-scale basis $(12,13)$, and it is also applicable in rather difficult operating conditions, such as studies in developing countries (14).

\section{METHODS AND SUBJECTS}

Term and preterm SGA and AGA infants, born in the neonatal unit of the Pediatric Department of San Paolo Hospital in Milan, were recruited throughout 1 y, starting November 2005, up to October 2006. For any SGA, two AGA, matched for GA $\pm 0.5 \mathrm{wk}$, were included. The study was approved by the review board of the Maternal-Infant Department at San Paolo Hospital. Parents gave their informed consent.

Entry criteria were: healthy and clinically stable (Apgar at $5 \mathrm{~min}>7$ ), and parental informed approval of infant participation obtained after birth. Only preterms $(<37$ th wk GA) clinically stable, admitted (if needed) into the intermediate care unit of our Department, and requiring at most parenteral glucose and amino acids (but not i.v. lipids) after birth were recruited for the study. As a consequence, our preterm infants' sample was constituted by infants with mild prematurity ( $>32$ nd wk GA in any case).

Exclusion criteria included habitual maternal alcohol consumption and smoking habits for the possible effects on infants' FA status that seem to be unrelated to being preterm or SGA $(15,16)$. Except cases requiring parenteral nutrient supplementations, all infants were fed their own mother's milk. The choice of a mother to formula feed was an exclusion criterion for the very early consequences of formula feeding on the FA status (17). The FA composition of Italian mothers' milk has been described by our group (18).

Definition and measurements. Maternal characteristics (age, prepregnancy body weight, weight increase in pregnancy, parity) were drawn from obstetrical charts. Maternal smoking habits and alcohol consumption were investigated through a questionnaire directly administered to mothers after delivery. Gestational age was assessed by the last menstrual period and confirmed by an ultrasound evaluation performed within the 20th wk GA. Maternal dietary habits were analyzed by means of a self-administered food frequency questionnaire (FFQ) consisting of 130 items specific for the examined population (pregnant women) related to the last $6 \mathrm{mos}$, designed according to Block (19), with a file for the frequency (daily, weekly, monthly) and standard measures indicating the eaten quantity, allowing for a semiquantitative estimation of the nutrient contribution. The FFQ forms were distributed to mothers after delivery, and were collected at discharge. For the data analysis, a software program linking the food frequency data with the nutrient database of food composition of the Italian Institute of Nutrition (20) has been developed according to indications on the correct use of FFQs for different study designs (21).
Infants were weighed naked within $30 \mathrm{~min}$ after delivery, and before the first feeding, by means of an electronic integrating scale (Sartorius, AG, Gottingen, Germany; precision $\pm 5.0 \mathrm{~g}$ ). Crown-to-heel length was measured on a recumbent infant board to the nearest millimeter by a trained operator using a Harpenden (UK) neonatometer. Cranial circumference was measured with a flexible narrow steel tape, which was applied firmly around the head above the supraorbital ridges. Infants were classified as AGA and SGA, respectively, according to Italian curves (22).

Laboratory analysis. FAs were analyzed in a drop of whole blood absorbed on a strip of chromatography paper by an innovative method validated for reproducibility (23), and already applied to studies in infants (16). Blood collected from a heel prick at the same time of the Guthrie neonatal screening test on d 4 of life was directly subjected to transmethylation for gas chromatography analysis. FA values were then expressed as FA weight percentages (\%) of total FA.

Statistical analysis. Descriptive data are shown as mean and SD values for population characteristics and dietary data. Because FAs have nonparametric within-group distribution, their values are described with median values and interquartile ranges. The SPSS package 14.0 for Windows (SPSS, Chicago, IL) was used for the statistical analyses. Categorical variables were evaluated with the $\chi^{2}$ test. ANOVA with the posthoc Student-Newman-Keuls test were used to evaluate between-group differences among the four study groups in case of parametric distribution. For FA values, with nonparametric distribution, the Kruskal-Wallis test and the posthoc Friedman test were used. Statistical significant level: $p<0.05$.

\section{RESULTS}

According to the study design, a total of 144 infants, born in the neonatal unit of the Pediatric Department at San Paolo Hospital in Milan and all breastfed, entered the study. According to GA and birth-weight they have been subdivided into 28 term SGA, 56 term AGA, 20 mildly preterm SGA, and 40 mildly preterm AGA. Maternal and anthropometrics characteristics are listed in Tables 1 and 2, respectively. Although no major differences in maternal characteristics were present (except for parity), weight, length, and cranial circumference were higher in term AGA infants versus the other three groups. Term SGA and preterm AGA showed quite similar values for weight, length, and head circumference.

Maternal dietary intakes are reported in Table 3. There were no statistically significant differences among the four groups as far as intakes of total energy, proteins, carbohydrates, and total fats were considered. However, a higher saturated fat intake has been found for mothers of term AGA in comparison with mothers of preterm AGA, whereas mothers of both SGA groups did not differ compared with any group.

Table 1. Maternal characteristics (mean $\pm S D$, when not differently indicated)

\begin{tabular}{|c|c|c|c|c|c|}
\hline & $\begin{array}{c}\text { Term } \\
\text { AGA (56) }\end{array}$ & $\begin{array}{c}\text { Term } \\
\text { SGA (28) }\end{array}$ & $\begin{array}{c}\text { Preterm } \\
\text { AGA }(40)\end{array}$ & $\begin{array}{l}\text { Preterm } \\
\text { SGA (20) }\end{array}$ & $p$ \\
\hline Maternal age $(y)$ & $32.3 \pm 4.3$ & $31.3 \pm 5.2$ & $31.7 \pm 4.8$ & $32.6 \pm 5.5$ & 0.749 \\
\hline Pre-pregnancy maternal weight $(\mathrm{Kg})$ & $61.0 \pm 8.7$ & $59.8 \pm 6.3$ & $59.1 \pm 7.6$ & $56.2 \pm 6.5$ & 0.127 \\
\hline Pregnancy weight increase $(\mathrm{Kg})$ & $11.8 \pm 3.9$ & $10.7 \pm 2.3$ & $10.5 \pm 2.3$ & $9.9 \pm 5.4$ & 0.130 \\
\hline Parity (n) $1, \geq 2$ & 29,27 & 20,8 & 13,27 & 10,10 & 0.018 \\
\hline
\end{tabular}

Table 2. Gestational age and neonatal characteristics at birth (mean $\pm S D)$

\begin{tabular}{|c|c|c|c|c|c|}
\hline & $\begin{array}{c}\text { Term } \\
\text { AGA (56) }\end{array}$ & $\begin{array}{c}\text { Term } \\
\text { SGA (28) }\end{array}$ & $\begin{array}{c}\text { Preterm } \\
\text { AGA (40) }\end{array}$ & $\begin{array}{c}\text { Preterm } \\
\text { SGA (20) }\end{array}$ & $p$ \\
\hline Gestational age (wk) & $38 \pm 0.9 *$ & $38 \pm 1.0^{*}$ & $35 \pm 1.0 \dagger$ & $35 \pm 1.0 \dagger$ & $<0.001$ \\
\hline Birth weight $(\mathrm{g})$ & $3222 \pm 412 *$ & $2430 \pm 261 \dagger$ & $2373 \pm 275 \dagger$ & $1865 \pm 285 \ddagger$ & $<0.001$ \\
\hline Birth length $(\mathrm{cm})$ & $49 \pm 1.0 *$ & $46 \pm 2.0 \dagger$ & $46 \pm 2.0 \dagger$ & $43 \pm 1.0 \ddagger$ & $<0.001$ \\
\hline Head circumference $(\mathrm{cm})$ & $34 \pm 1.0^{*}$ & $32 \pm 1.0 \dagger$ & $32 \pm 1.0 \dagger$ & $30 \pm 1.0 \ddagger$ & $<0.001$ \\
\hline
\end{tabular}

Different superscripts indicate significant between-group differences $(p<0.05)$. 
The percentage values of the major classes of FA in whole blood lipids are reported in Table 4. Differences were present for total monounsaturated, lower in the case of term SGA, and total n-3 PUFA, lower in the case of preterms, respectively. As concerns ratios between n-3 and n-6 FA, they were higher in term groups, in parallel with higher total $n-3$ concentrations.

Table 5 shows the percentage values of the main PUFA. The precursors of the two polyunsaturated FA series, LA, for the $n-6$, and ALA, for the n-3 series, respectively, were progressively higher in the sequence from term AGA to preterm groups, whereas the inverse trend occurred for the major LCPUFA, less markedly in the n- 6 series, for di-homogamma-linolenic acid (20:3 n-6), and more markedly for DHA, the end-product of the n-3 series. Peculiarly, eicosa- pentaenoic acid (20:5 n-3) reached the highest level in term SGA. Term infants showed a trend toward higher AA levels compared with preterms.

Finally, in Table 6 the most relevant product/precursor ratios of both the n- 6 and the n-3 series are reported, which are representative of the metabolic steps involved in PUFA biosynthesis, regulated by alternating desaturase and elongase enzymes. In particular, the AA/LA ratio, and then the conversion rate of the essential LA to AA, the main n-6 LCPUFA, showed higher levels in term AGA and lower in term SGA infants and preterm groups, suggesting a reduction of the $\Delta 6$ desaturation activity. In the case of the n-3 series the corresponding product/precursor ratio, EPA/ALA, is higher in both term groups, even if for term SGA infants the median value is

Table 3. Maternal dietary intakes (mean $\pm S D$ )

\begin{tabular}{lcccc}
\hline & Term AGA (51) & Term SGA (23) & Preterm AGA (33) & Preterm SGA (15) \\
\hline kcal & $2268 \pm 800$ & $2482 \pm 739$ & $2035 \pm 616$ & $2253 \pm 756$ \\
Protein\% & $15.4 \pm 2.4$ & $15.6 \pm 1.8$ & $15.9 \pm 3.3$ & $17.0 \pm 2.8$ \\
Carbohydrate\% & $51.8 \pm 7.5$ & $52.2 \pm 9.4$ & $55.4 \pm 6.2$ & $50.7 \pm 10.3$ \\
Fat\% & $33.5 \pm 6.6$ & $33.5 \pm 8.0$ & $31.0 \pm 4.7$ & $34.7 \pm 7.8$ \\
SFA\% & $12.2 \pm 3.3 *$ & $11.8 \pm 3.2$ & $10.3 \pm 1.9 \dagger$ & 0.262 \\
MUFA\% & $14.7 \pm 3.7$ & $15.3 \pm 4.7$ & $14.3 \pm 3.2$ & 0.223 \\
PUFA\% & $4.5 \pm 1.7$ & $4.5 \pm 1.6$ & $4.4 \pm 1.0$ & 0.024 \\
\hline
\end{tabular}

Different superscripts indicate significant between-group differences $(p<0.05)$.

SFA, saturated fatty acids; MUFA, monounsaturated fatty acids; PUFA, polyunsaturated fatty acids.

Table 4. Major fatty acids families (median, interquartile range) as fatty acid \% and ratio between total n-3 and n-6 fatty acids in infants' blood

\begin{tabular}{lcccc}
\hline & Term AGA (56) & Term SGA (28) & Preterm AGA (40) & Preterm SGA (20) \\
\hline SFA & $46,44-48$ & $47,45-49$ & $46,45-47$ & $45,43-49$ \\
MUFA & $25,24-27^{*}$ & $23,21-25 \dagger$ & $25,24-27^{*}$ & $25,23-28^{*}$ \\
PUFA & $27,26-29$ & $29,25-30$ & $27,25-28$ & $27,24-29$ \\
Total n-6 & $22,21-24$ & $23,21-24$ & $22,21-23$ & $22,21-24$ \\
Total n-3 & $4.7,4.0-5.3^{*}$ & $4.9,4.5-5.4^{*}$ & $3.8,3.3-4.3 \dagger$ & 0.002 \\
n-3/n-6 & $0.20,0.18-0.23^{*}$ & $0.21,0.19-0.23^{*}$ & $0.16,0.14-0.18 \dagger$ & 0.115 \\
\hline
\end{tabular}

Different superscripts indicate significant between-group differences $(p<0.05)$.

SFA, saturated fatty acids; MUFA, monounsaturated fatty acids; PUFA, polyunsaturated fatty acids.

Table 5. Major polyunsaturated fatty acids (median, interquartile range) as fatty acid \% in infants' blood

\begin{tabular}{|c|c|c|c|c|c|}
\hline & Term AGA (56) & Term SGA (28) & Preterm AGA (40) & Preterm SGA (20) & $p$ \\
\hline C18:2 n-6 & $4.3,3.4-5.1 *$ & $5.0,4.4-6.5 \dagger$ & $6.2,4.6-7.2 \dagger$ & $5.9,5.1-7.9 \dagger$ & $<0.001$ \\
\hline$C 20: 3 n-6$ & $1.7,1.6-2.0^{*}$ & $1.6,1.3-1.9$ & $1.5,1.3-1.7 \dagger$ & $1.4,1.2-1.6 \dagger$ & $<0.001$ \\
\hline C20:4 n-6 & $12.9,11.8-14.1$ & $12.9,11.4-13.7$ & $12.1,10.8-13.5$ & $12.1,10.3-13.2$ & 0.058 \\
\hline C22:4 n-6 & $2.1,1.8-2.5^{*}$ & $2.2,1.8-2.6^{*}$ & $1.9,1.7-2.2 \dagger$ & $1.7,1.2-2.3 \dagger$ & 0.006 \\
\hline$C 22: 5 n-6$ & $1.0,0.8-1.2 *$ & $1.0,0.8-1.3^{*}$ & $0.81,0.65-1.15 \dagger$ & $0.73,0.52-0.99 \dagger$ & $<0.001$ \\
\hline C18:3 n-3 & $0.04,0.03-0.06^{*}$ & $0.15,0.09-0.30 \dagger$ & $0.19,0.12-0.32 \dagger$ & $0.28,0.18-0.46 \dagger$ & $<0.001$ \\
\hline C20:5 n-3 & $0.18,0.14-0.24 *$ & $0.38,0.19-1.07 \dagger$ & $0.17,0.14-0.26^{*}$ & $0.23,0.15-0.31^{*}$ & $<0.001$ \\
\hline $\mathrm{C} 22: 5 \mathrm{n}-3$ & $0.40,0.30-0.53$ & $0.46,0.29-0.63$ & $0.23,0.38-0.71$ & $0.59,0.23-0.71$ & 0.608 \\
\hline $\mathrm{C} 22: 6 \mathrm{n}-3$ & $3.9,3.4-4.6^{*}$ & $3.7,2.9-4.1^{*}$ & $2.8,2.3-3.2 \dagger$ & $2.4,1.8-3.2 \dagger$ & $<0.001$ \\
\hline
\end{tabular}

Different superscripts indicate significant between-group differences $(p<0.05)$.

Table 6. Major product/precursor ratios of $n-6$ and $n-3$ series (median, interquartile range) in infants' blood

\begin{tabular}{lccccc}
\hline & Term AGA (56) & Term SGA (28) & Preterm AGA (40) & Preterm SGA (20) \\
\hline AA/LA & $2.9,2.3-3.7^{*}$ & $2.4,1.9-2.9 \dagger$ & $2.0,1.5-2.6 \dagger$ & $2.1,1.2-2.5 \dagger$ \\
EPA/ALA & $4.2,2.6-5.7^{*}$ & $1.9,0.9-9.9^{*}$ & $0.8,0.4-1.9 \dagger$ & $0.9,0.3-1.2 \dagger$ \\
DHA/ALA & $91,60-118^{*}$ & $18,10-39 \dagger$ & $12,7-23 \dagger$ & $10,4-16 \dagger$ & $<0.001$ \\
AA/EPA & $72,50-89^{*}$ & $29,11-67 \dagger$ & $70,48-90^{*}$ & $<1,41-83$ & $<0.001$ \\
\hline
\end{tabular}

Different superscripts indicate significant between-group differences $(p<0.05)$. 
quite lower, in face of a wide interquartile range in association with maximal EPA concentrations. On the other hand, the index of the cumulative n-3 FA metabolic pathway, expressed by the DHA/ALA ratio, is quite higher in the term AGA group compared with all the other three groups. Therefore, the production of metabolic end products of the n-3 series appears to be interrupted at the level of EPA for term SGA, in which the ratio between the two 20 carbon PUFA, AA and EPA, is significantly lower versus the other three groups.

\section{DISCUSSION}

We have evaluated the whole blood FA profiles in healthy infants, born at term and preterm, according to a matched study design comparing AGA and SGA infants in both groups, respectively. Because blood sampling was obtained at d 4, even considering the possible differences in the LCPUFA content in milk of mothers with preterm delivery, as found in cases of severe, and not mild, prematurity (24), the amount of milk consumed by the infant during the first days of life would have just a minimal impact on his LCPUFA status, at the point to explain differences in the FA pattern. Moreover, it has already been demonstrated that the relationships between LCPUFA concentrations at birth and postnatal changes during the first 6 wks after birth are just partly dependent on the composition of the supplied milk (25).

To our knowledge this is the first matched study on the FA status of term and preterm infants, subdivided into AGA and SGA, with four defined groups, taking into consideration also the nutrient composition of maternal diet. In addition, the FA analysis has been carried out on whole blood, inclusive of both circulating and membrane cell lipids, and therefore mostly representative of the FA status in the body on a sample out of any possible contamination from placental blood (11), reflecting both maternal passage and fetal/neonatal synthesis.

The analysis of the FA composition shows that essential FA, LA and ALA, are higher, whereas the major metabolic products, AA and DHA, together with the total n-3 PUFA are lower in preterm AGA and SGA, in comparison with the term groups, suggesting that mostly gestational age, i.e. the duration of the intrauterine life, affects blood PUFA levels. On the other side, if we consider the product/precursor ratios, it is evident that all the conversion rates are significantly higher in term AGA infants versus all the other three groups, indicating that optimal biosynthetic performances and/or complete metabolic transfers from the maternal compartment to infants may take place only in case of term delivery and adequate intrauterine growth. These between-neonate differences are not associated with differences in PUFA maternal dietary intakes. Indeed term AGA infants represent the golden standard as far as the FA pattern is considered. Levels of essential FA precursors are lower than those in the other groups. The higher levels of DHA in term AGA indicate that most transfer and incorporation of this FA in cells and tissues takes place during the last weeks of pregnancy in the normally growing infants. On the basis of our findings, however, it is not possible to speculate whether the different distributions of PUFA of the two series are associated to major changes in synthesis within fetal compartments and/or transfer from the maternal FA pool at the placenta level.

Compared with term AGA, term SGA infants have higher levels of $18 \mathrm{C}$ carbon precursors but similar concentrations of n-6 LCPUFA and total n-3 FA. As regards EPA levels, they are higher in term SGA than in term AGA. We may speculate that the excess of EPA in term SGA could be representative of a block behind the last metabolic steps in DHA synthesis taking place in the last weeks of term pregnancies. An alternative interpretation is that the lower rate of body growth in SGA versus AGA results in lower incorporation in cells and tissues, especially in phospholipid pools, of DHA, the end product of the $n-3$ series. Whatever the underlying mechanism for the elevation of EPA, as a consequence, the AA/EPA ratio is quite lower in term SGA, compared in particular with the two AGA groups. To what extent this reduction of AA relative to its analogous 20C of the n-3 series (EPA) could contribute to some unfavorable conditions within this group $(26,27)$ is unknown. The peculiarity of the FA pattern of term SGA infants is further emphasized by the observation of lower levels of total monounsaturated fats. Although the biologic relevance of this observation is unclear, we could speculate that SGA infants near the term could increase the relative use of monoenes for energy production, considering the reduced fat stores in intrauterine growth retardation coupled with a less efficient energy production from glucose $(28,29)$. The high EPA levels within term SGA seem to be apparently in contrast with Rump et al., (10) who found that EPA at birth was not related to weight for gestational age at birth, while a trend toward lower levels of circulating monounsaturated FA in parallel with lower birth weight was present. Although in Rump's study FA were analyzed in cord blood phospholipids, we analyzed whole blood-inclusive of circulating cell membranes, where LCPUFA are more concentrated-collected at d 4 with the Guthrie test.

Our data are consistent with findings from the studies with stable isotopes in IUGR infants showing specific impairments in the final biosynthetic pathways leading to the synthesis of LCPUFA, particularly DHA $(8,9)$. Compared with these studies, the new information from our study includes the lack of associations with major differences in PUFA maternal dietary intakes, the high EPA-low monounsaturated FA levels in term SGA, and that even mild prematurity (that is, an average gestational age of around $35 \mathrm{wks}$ ) is associated with lower LCPUFA levels, particularly DHA, compared with term infants. Accordingly, maximal blood accumulation of the n-3 LCPUFA seems to occur in the last weeks of pregnancy, leading to DHA and EPA peaking in term AGA and SGA infants, respectively. Although the DHA peaking in term infants represent the physiologic situation, the EPA peaking in term SGA could be expression of their "unphysiological" metabolic condition. Therefore, although a dietary supply of DHA is advisable, common fish oils, inclusive of both EPA and DHA (and in general with higher EPA concentrations compared with DHA), could not be the best choice for this group. Investigators should elucidate in future studies the correlations between blood and tissue FA profiles (in particular of the CNS) and the associations between biochemical 
markers and tissue functions (considering the complications of reduced fetal growth at the neurovascular level) (27), to optimize indications of dietary fat requirements in preterm and term infants, based on being either AGA or SGA, respectively.

\section{REFERENCES}

1. Dijck-Brouwer DA, Hadders-Algra M, Bouwstra H, Decsi T, Boehm G, Martini IA, Boersma ER, Muskiet FA 2005 Lower fetal status of docosahexaenoic acid, arachidonic acid and essential fatty acids is associated with less favorable neonatal neurological condition. Prostaglandins Leukot Essent Fatty Acids 72:21-28

2. Haggarty P, Ashton J, Joynson M, Abramovich DR, Page K 1999 The effect of maternal polyunsaturated fatty acid concentration on transport by the human placenta. Biol Neonate 75:350-359

3. Haggarty P 2004 Effect of placental function on fatty acid requirements during pregnancy. Eur J Clin Nutr 58:1559-1570

4. Carnielli VP, Wattimena DJ, Luijendijk IH, Boerlage A, Degenhart HJ, Sauer PJ 1996 The very low birth weight premature infant is capable of synthesizing arachidonic and docosahexaenoic acids from linoleic and linolenic acids. Pediatr Res 40:169-174

5. Crawford MA, Hassam AG, Williams SC 1976 Essential fatty acids and brain growth. Lancet 308:452-453

6. Koletzko B, Agostoni C, Carlson SE, Clandinin T, Hornstra G, Neuringer M, Uauy R, Yamashiro Y, Willatts P 2001 Long chain polyunsaturated fatty acids (LC-PUFA) and perinatal development. Acta Paediatr 90:460-464

7. Cetin I, Giovannini N, Alvino G, Agostoni C, Riva E, Giovannini M, Pardi G 2002 Intrauterine growth restriction in associated with changes in polyunsaturated fatty acid fetal-maternal relationships. Pediatr Res 52:750-755

8. Uauy R, Mena P, Wegher B, Nieto S, Salem N Jr 2000 Long chain polyunsaturated fatty acid formation in neonates: effect of gestational age and intrauterine growth. Pediatr Res 47:127-135

9. Llanos A, Lin Y, Mena P, Salem N Jr, Uauy R 2005 Infants with intrauterine growth restriction have impaired formation of docosahexaenoic acid in early neonatal life: a stable isotope study. Pediatr Res 58:735-740

10. Rump P, Mensink RP, Kester AD, Hornstra G 2001 Essential fatty acid composition of plasma phospholipids and birth weight: a study in tern neonates. Am J Clin Nutr 73:797-806

11. Hall JM, Lingenfelter P, Adams SL, Lasser D, Hansen JA, Bean MA 1995 Detection of maternal cells in human umbilical cord blood using fluorescence in situ hybridization. Blood 86:2829-2832

12. Baylin A, Kim MK, Donovan-Palmer A, Siles X, Dougherty L, Tocco P, Campos H 2005 Fasting whole blood as a biomarker of essential fatty acid intake in epidemiologic studies: comparison with adipose tissue and plasma. Am J Epidemiol 162:373-381

13. Risé P, Eligini S, Ghezzi S, Colli S, Galli C 2007 Fatty acid composition of plasma, blood cells and whole blood: relevance for the assessment of the fatty acid status in humans. Prostaglandins Leukot Essent Fatty Acids 76:363-369
14. Agostoni C, Giovannini M, Sala D, Usuelli M, Livio L, Francescato G, Braga M, Riva E, Martiello A, Colombo C, Marangoni F, Galli C 2007 Double-blind, placebocontrolled trial comparing effects of supplementation of two micronutrient sprinkles on fatty acid status in Cambodian infants. J Pediatr Gastroenterol Nutr 44:136-142

15. Smuts CM, Tichelaar HY, Dhansay MA, Faber M, Smith J, Kirsten GF 1999 Smoking and alcohol use during pregnancy affects preterm infants' docosahexaenoic acid (DHA) status. Acta Paediatr 88:757-762

16. Agostoni C, Galli C, Riva E, Colombo C, Giovannini M, Marangoni F 2005 Reduced docosahexaenoic acid synthesis may contribute to growth restriction in infants born to smoking mothers. J Pediatr 147:854-856

17. Sanjurjo P, Rodriguez-Alarcon J, Rodriguez-Soriano J 1988 Plasma fatty acid composition during the first week of life following feeding with human milk or formula. Acta Paediatr Scand 77:202-206

18. Marangoni F, Agostoni C, Lammardo AM, Giovannini M, Galli C, Riva E 2000 Polyunsaturated fatty acid concentrations in human hindmilk are stable throughout 12-months of lactation and provide a sustained intake to the infant during exclusive breastfeeding: an Italian study. Br J Nutr 84:103-109

19. Block G, Hartman AM, Dresser CM, Carroll MD, Gannon J, Gardner L 1986 A data-based approach to diet questionnaire design and testing. Am J Epidemiol 124:453-469

20. Italian Institute of Nutrition 1998 Food Composition Tables. Rome: Italian Institute of Nutrition

21. Cade J, Thompson R, Burley V, Warm D 2002 Development, validation and utilisation of food-frequency questionnaires-a review. Public Health Nutr 5:567-587

22. Gagliardi L, Macagno D, Pedrotti D, Coraiola M, Furlan R, Agostinis L, Milan S 1999 Weight, length and head circumference at birth of a North-eastern Italian population. Report of the ad hoc committee of the Italian society of neonatology. Riv Ital Pediatr 25:159-169 (Italian Journal of Pediatrics)

23. Marangoni F, Colombo C, Galli C 2004 A method for the direct evaluation of the fatty acid status in a drop of blood from a fingertip in humans: applicability to nutritional and epidemiological studies. Anal Biochem 326:267-272

24. Kovács A, Funke S, Marosvölgyi T, Burus I, Decsi T 2005 Fatty acids in early human milk after preterm and full-term delivery. J Pediatr Gastroenterol Nutr 41:454-459

25. Guesnet P, Pugo-Gunsam P, Maurage C, Pinault M, Giraudeau B, Alessandri JM, Durand G, Antoine JM, Couet C 1999 Blood lipid concentrations of docosahexaenoic and arachidonic acids at birth determine their relative postnatal changes in term infants fed breast milk or formula. Am J Clin Nutr 70:292-298

26. Carlson SE, Werkman SH, Peeples JM, Cooke RJ, Tolley EA 1993 Arachidonic acid status correlates with first year growth in preterm infants. Proc Natl Acad Sci USA 90:1073-1077

27. Crawford M 2000 Placental delivery of arachidonic and docosahexaenoic acids: implications for the lipid nutrition of preterm infants. Am J Clin Nutr 71:275S-284S

28. Pardi G, Cetin I, Marconi AM, Lanfranchi A, Bozzetti P, Ferrazzi E, Buscaglia M, Battaglia FC 1993 Diagnostic value of blood sampling in fetuses with growth retardation. N Engl J Med 328:692-696

29. Sparks JW, Ross JC, Cetin I 1998 Intrauterine growth and nutrition. In: Polin RA, Fox WW (eds) Fetal and Neonatal Physiology. 2nd ed. Oxford: WB Saunders, pp 267-289 